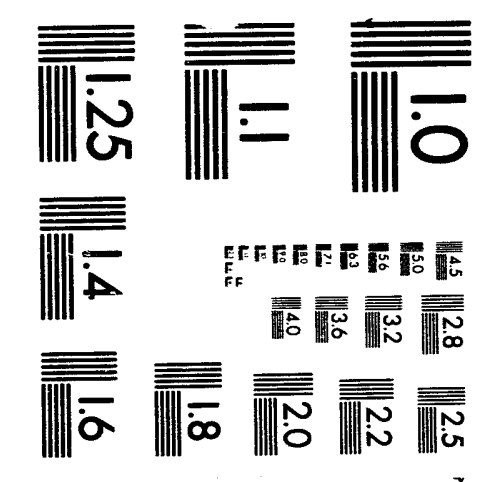



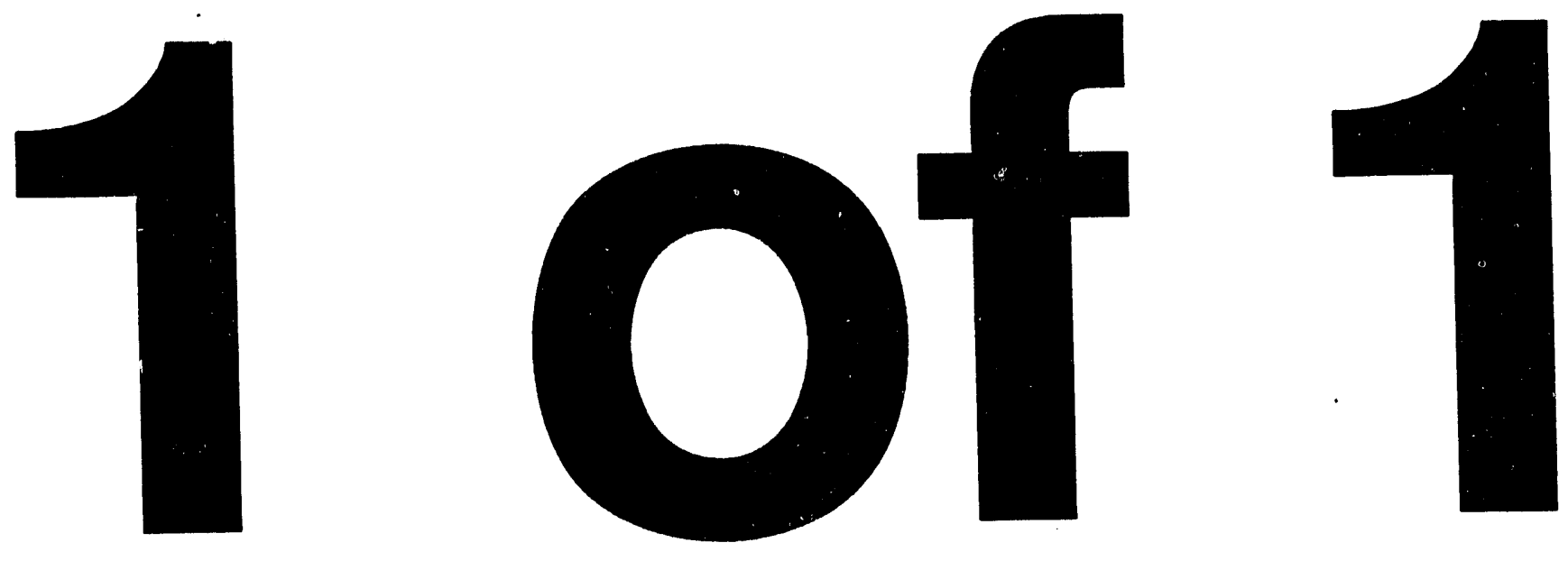
Title: Long-Range Alpha Detector (LRAD) Sensitivity to Beta Contamination and Soil Moisture

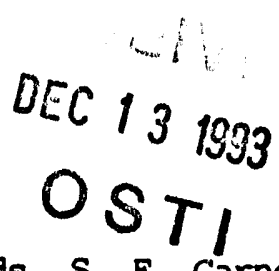

J. D. Johnson, K. S. Allander, J. A. Bounds, S. E. Garner, J. P. Johnson, D. W. MacArthur

IEEE 1993 Nuclear Science Symposium

October 31-November 6, 1993

San Eransisco, California

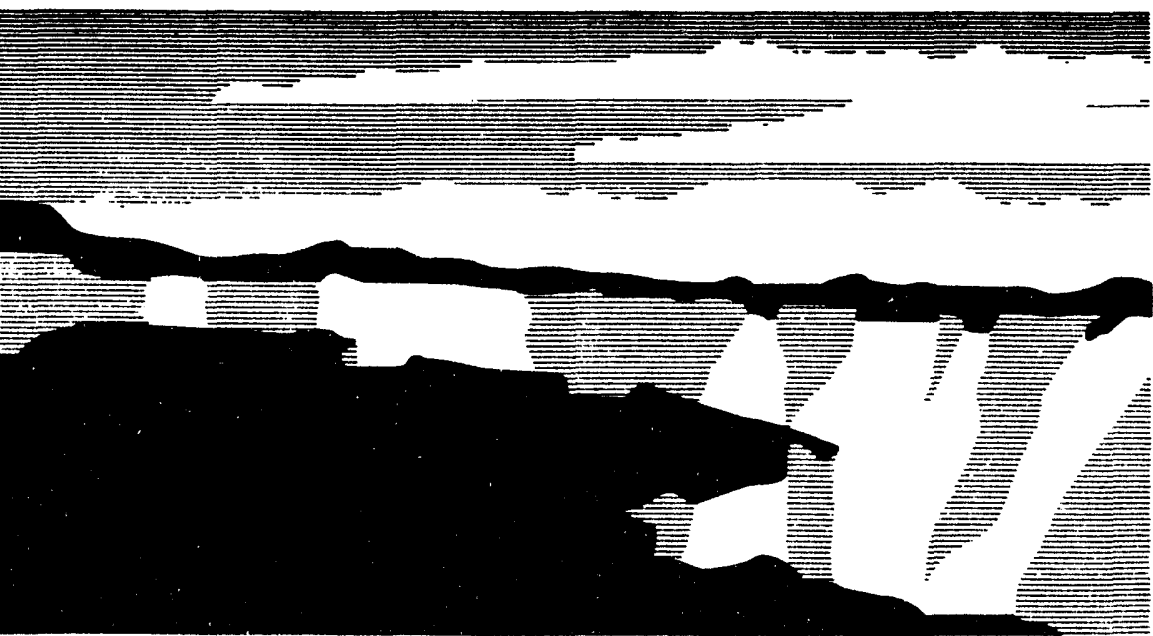

Los Alamos National Laboratory, en affirmative actionvequal opportunity empldyer, is operaied by the University of California tor the U.S. Depantment of Einergy under contract W-7405-ENG-38. By scceptance of this articts, the publisher recognizes that the U.S. Government reteins a nonexclusive, royality-free license to publish or reproduce the published form of this contribution, or to allow others to do so, for U.S. Government puppees. The Los Aamos National Laboratory

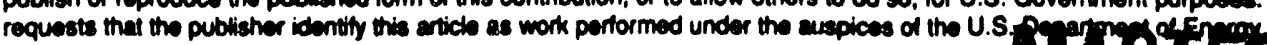




\title{
Long-Range Alpha Detector (LRAD) Sensitivity to Beta Contamination and Soil Moisture
}

\author{
J. D. Johnson, K. S. Allander, J. A. Bounds, S. E. Garner, \\ J. P. Johnson, and D. W. MacArthur \\ Los Alamos National Laboratory, Los Alamos, NM 87545
}

\section{Abstract}

Long-Range Alpha Detector (LRAD) systems are designed to monitor alpha contamination by measuring the number of ions in the air. Alpha particles are a form of ionizing radiation and a typical 5-MeV alpha particle will create about 150,000 ion pairs in air. Recent tests have been performed to determine the sensitivity of LRAD systems to beta contamination and soil moisture levels. These results and the general technology are discussed in this paper.

\section{INTRODUCTION}

Traditional alpha detectors are designed to detect alpha particles directly and must be passed slowly within $3 \mathrm{~cm}$ of an alpha source to operate effectively. Because LRAD detectors collect the ions created from alpha interactions with air, they are better able to monitor equipment and complex surfaces and can be operated at a much greater distance from an alpha source than traditional alpha detectors. Furthermore, because LRAD detectors remain stationary during monitoring, they are less subject to operator error than traditional alpha detectors. The LRAD Surface Soil Monitor (SSM) and the LRAD Sample Monitor are based on an electrostatic design, where the ions created by the interaction of alphas with air are collected using an electrostatic field. These detectors have been used to characterize radioactive contamination at Los Alamos National Laboratory (LANL), Fernald, and Grand Junction. LRAD object monitors are based on an airflow design. As opposed to the electrostatic design, these detectors collect air ions using fans to draw the air through the detector. These detectors are able to measure contamination in confined areas that are not easily measured by traditional alpha detectors. Various LRAD object monitors have been successfully demonstrated at LANL, Savannah River, and Rocky Flats. [16]. This paper will discuss some recent tests made using LRAD Surface Soil Monitors.

\section{SURFACE SOIL MONITOR DESIGN}

The basic design for the LRAD SSM shown in Fig. 1 consists of a grounded enclosure and a high-voltage guard plane and signal plane. The geometry of this detector is such that all of the alphas from the ground stop before reaching the signal plane, and ions of one polarity are collected on the signal plane. The alpha contamination can then be calculated by directly scaling the measured current from the signal plane. Because the current response of an LRAD detector is a linear function of the activity of the sample being monitored, it is possible to cross-calibrate an LRAD to sources of know activity.

An electrometer sensitive to the femtoamp $\left(10^{-15} \mathrm{~A}\right)$ level is used to measure the current from the signal plane, and the guard and signal planes are kept at $300 \mathrm{~V}$ with a dc battery. By Ohm's Law, if the signal plane is maintained at $300 \mathrm{~V}$ and the leakage current is required to be $<100 \mathrm{fA}$, the insulation resistance between the signal plane and the ground must be at least $3 \times 10^{15} \Omega$ [2]. By using a guard plane, the only leakage current passing through the electrometer is due to the offset voltage of the electrometer. If the offset voltage in the electrometer is $3 \mathrm{mV}$, then the insulation resistance between the signal plane and the ground needs to be $3 \times 10^{10} \Omega$. The effect of the guard plane is to help control leakage current and. therefore, improve the sensitivity of the detector.

The normal operating procedure for using an SSM' is to place the detector directly on soil to be monitored. The detector and soil form a closed box that is grounded. Three different sized detectors have been used for monitoring soil surface contamination. Tests have shown that the response time, or time required to make a statistically significant measurement, scales as the volume of the detector being used. The source strength per unit area that can be detected seems to be fairly consistent for all three detectors.

\section{BetA ATtENUATION TESTS}

A series of tests was carried out at LANL to determine the effects of beta contamination on typical LRAD $\mathrm{m}$ :asurer For these tests the Large SSM $\left(1-\mathrm{m}^{2}\right.$ footprini: an smal! SSM $\left(0.25-\mathrm{m}^{2}\right.$ footprint) were used to m contamination and source strength for alpha $a_{i}{ }^{-1}$ et $x$ snitte; The soil contained only natural backgr wid liveis of contamination. The alpha emitter used v/as 9240 , $80 \mathrm{f}$ - dom

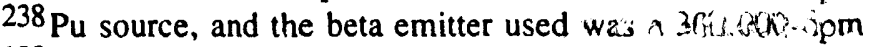
${ }^{198} \mathrm{Au}$ source. The measured signals minui, backz: ourid, fo: the Large SSM were $35,924 \mathrm{fA}$ for the $238 \mathrm{Am}$, $5 \mathrm{C}_{1}$, for the

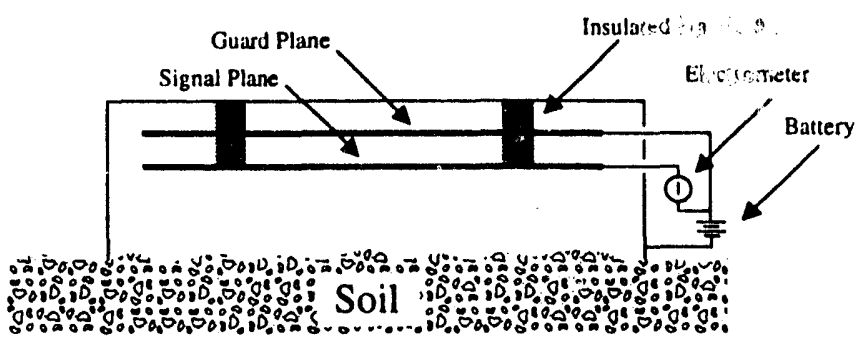

Fig. 1. Schematic view of the LRAD Surface Soil Monitor. 
$198 \mathrm{Au}$, and $697 \mathrm{fA}$ for the soil. The Small SSM tests included both soil on a tray and outdoor soil in situ. The measured signals minus backgrounds for the Small SSM wern $35.832 \mathrm{fA}$ for the ${ }^{238} \mathrm{Pu}, 341 \mathrm{fA}$ for the ${ }^{198} \mathrm{Au}, 271 \mathrm{fA}$ for the soil on the tray, and $959 \mathrm{fA}$ for the out door soil. Measurements were first made with these three sources ( ${ }^{238} \mathrm{Pu},{ }^{198} \mathrm{Au}$, and soil) alone. then various layers of Mylar were added to cover each source. The sheets of Mylar were 0.25 mils thick, coated with $400 \AA$ of aluminum on each side.

The results for the Large SSM are shown in Fig. 2, and the results for the Small SSM are shown in Fig. 3. These results are given in terms of percent signal, normalized to ( $\mathrm{Sl}-\mathrm{Bkg}$ ) for each source, where $S 1$ is the signal of the source measured with no Mylar. By plotting the results, which varied greatly in terms of absolute current measured, in terms of \% signal, it can be shown that the layers of Mylar attenuated the alpha particles significantly while having little effect on the beta particles. The results of these Mylar tests indicate that the signal from the soil is consistent with that of the alpha source $\left({ }^{238} \mathrm{Pu}\right)$, rather than the beta source $\left({ }^{198} \mathrm{Au}\right)$. This can be seen in Fig. 2 and Fig. 3, where the additional layers of Mylar caused the \% signal to fall off at roughly the same rate for the alpha source as the soil.

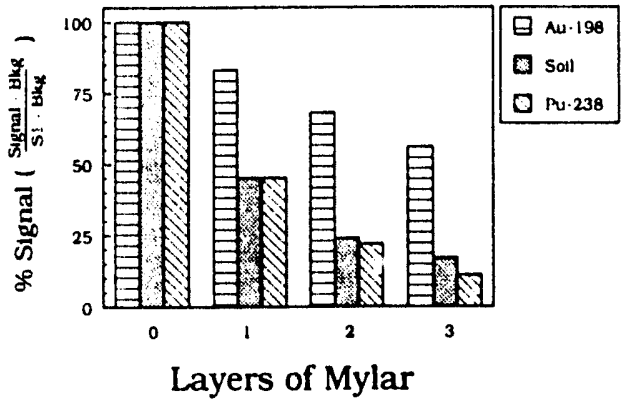

Fig. 2. Results of the Mylar tests using the Large SSM. By normalizing each sample to the measurement which has no Mylar, the results are plotted in terms of \% signal from 0 to $100 \%$.

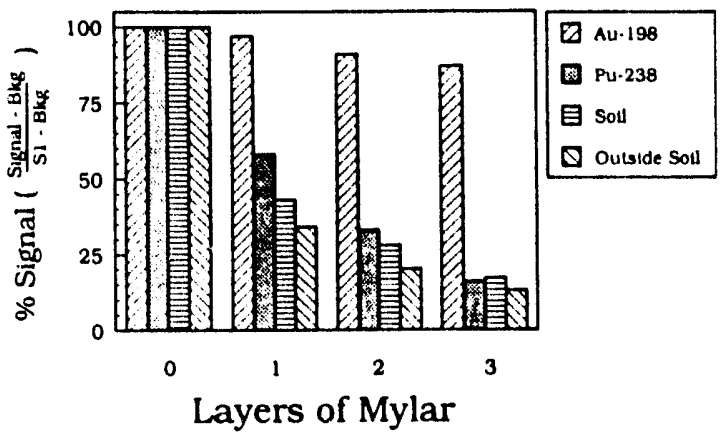

Fig. 3. Results of the Mylar tests using the Small SSM. By normalizing each sample to the measurement which has no Mylar, the results are plotted in terms of \% signal from 0 to $100 \%$.
Both the alpha and beta emitters had roughly the same source strength in terms of disintegrations per minute $(\mathrm{dpm})$ : However, the alpha source was detected much more strongly than the beta source. This is to be expected from the range and energy of alphas vs those of betas. The LRAD SSM is capable of measuring both alpha and beta contamination; however, the relative contribution of each will depend upon the particular source being measured. The error bars in these tests were typically $5 \%$ or less, except for the outdoor soil, where they were 5-10\%. These tests have shown that the electrostatic LRAD design is sensitive to both alpha and beta contamination in field measurements of soil. However, the alpha contamination levels are such that they overwhelm the beta readings for typical soil.

\section{SOIL MOISTURE TESTS}

To better model field conditions where wet soil is involved and to get a better idea of the sensitivity of the LRAD Surface Soil Monitors, soil moisture levels have been measured at LANL. Ordinary soil taken from outdoors was heat dried and used as the $0 \%$ moisture sample. Water was added, and the sample was weighed to determine its percent-water content. The LKAD hand-held SSM was used for this test, and the results can be extended to show that the LRAD electrostatic monitor design can accurately measure soil with $>20 \%$ water content. This level of moisture was beyond the saturation point for the sample of soil used. These results tend to indicate that an LRAD electrostatic monitor design will be able to monitor soil contamination levels under most reasonable field conditions. The results of these tests are shown in Figs. 4 and 5 , where the error bars were typically $5-10 \%$. The fluctuations in the data increased as the water content increased, indicating that the time required to assay soil with a higher water content would increase as well. The actual on-line current measurements for this test is shown in Fig. 6.

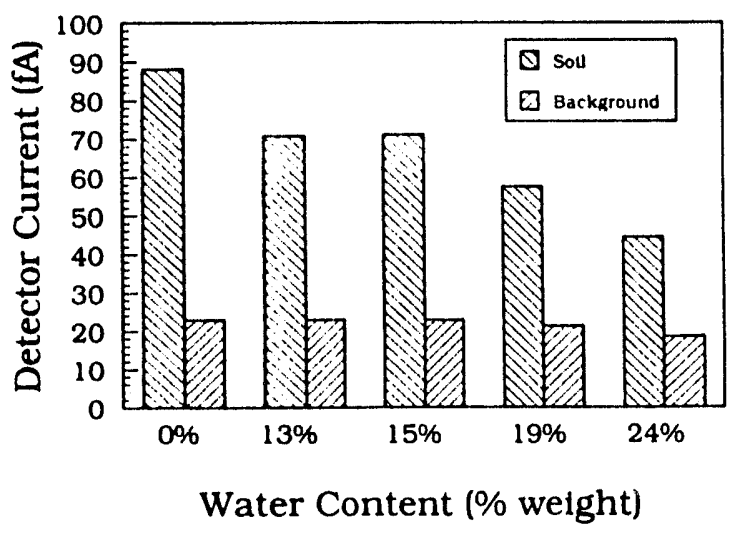

Fig. 4. Background levels (with no sample) and soil measurements are shown for soil moisture tests using the Handheld SSM. The water content is given as a percent of the total weight of the sample. 


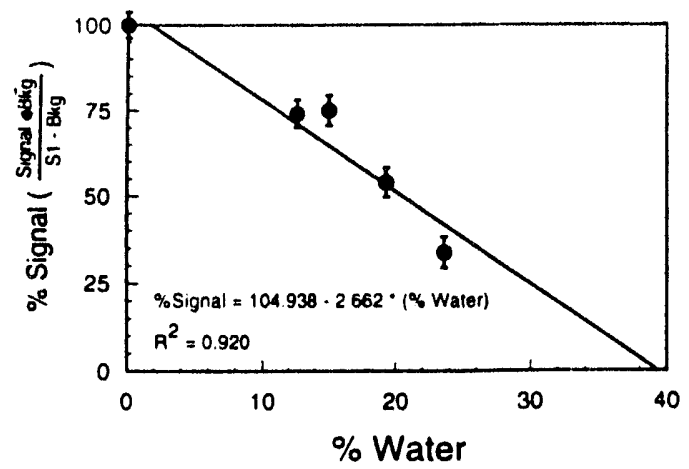

Fig. 5. Results of the water content measurements shown in terms of \% signal. A straight line curve-fit with $R^{2}=0.920$ has been made to show the tendency of the signal to drop oif as more water is added to the soil.

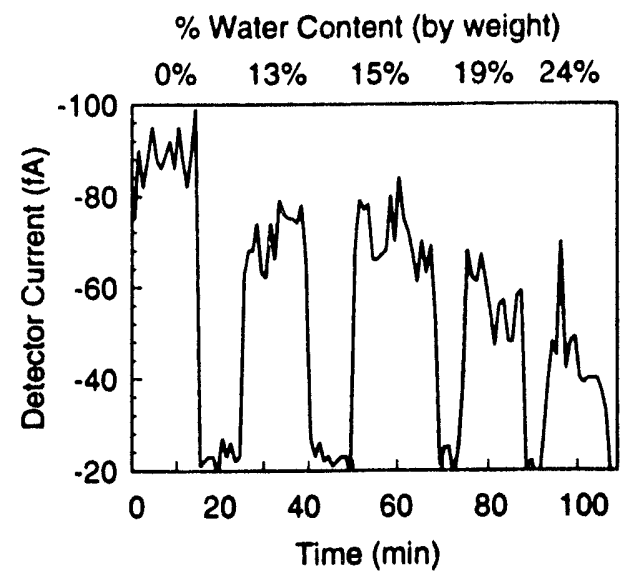

Fig. 6. On-line current measurements for water content measurements using the LRAD Hand-held SSM. The water content is given as a percent of the total weight of the sample.

\section{CONCLUSIONS}

Along with the various field tests of the LRAD electrostatic monitor design, these tests indicate that LRAD monitors can be used for many applications. An LRAD Soil Sample Monitor has been developed that can be used to monitor soil samples in the field. Tests at LANL have shown that the LRAD Soil Sample Monitor can assay soil in just $30 \mathrm{~s}$, and for contamination levels of $<100 \mathrm{dpm}$ above background levels. The LRAD Soil Sample Monitor is being used to determine what correlations exist between detector response and various environmental factors.

Other LRAD electrostatic monitor designs, currently in the conceptual phase, include solid waste monitors, conveyor-belt based monitors, and liquid stream monitors.

\section{REFERENCES}

[1] D. W. MacArthur et. al., "LRAD Surface Monitors," Los Alamos National Laboratory report LA-12524-MS (1993).

[2] D. W. MacArthur et. al., "LRAD Alpha Monitoring at Fernald. Los Alamos National Laboratory report LA-UR-92-3032 (1992).

[3] D. W. MacArthur et. al., "Alpha Contamination Monitoring of Surfaces, Objects, and Enclosed Areas," Los Alamos National Laboratory report LA-UR-92-3433 (1992).

[4] D. W. MacArthur et. al., "TA-06 Alpha Monitoring Results," Los Alamos National Laboratory memorandum N2 92:852MMC to C. Rofer (July 1992).

[5] J. D. Johnson, "Evaluation of LRAD Soil Sample Monitor," Los Alamos National Laboratory memorandum N2-93:1134JDJ to Paul Aamodt (September 1993).

[6] S. E. Garner, "HS-1 Alpha Monitoring Results," Los Alamos National Laboratory memorandum N2-93:1147SEG to Peter Ververka (October 1993). 



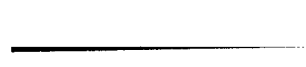

